\title{
Enteric vaccines for the developing world: a challenge for mucosal immunology
}

\author{
C Czerkinsky $^{1}$ and J Holmgren ${ }^{2}$
}

Enteric infections kill approximately two million children under the age of 5 in developing countries and cause more than four billion disease episodes worldwide each year. In addition, these diseases affect the growth, cognitive function, and quality of life negatively. There is an urgent need for vaccines that induce effective and long-lasting intestinal immunity against diarrheal infections, especially during infancy and early childhood. Yet, most vaccines available are formulated on an empirical basis. To date, arguably, vaccines have done more for immunologists than immunologists have done for vaccines.

\section{MUCOSAL VACCINES-AN UNMET NEED}

The tide is changing, however, as immunologists translate new discoveries into vaccines against diseases such as cholera, rotavirus, and typhoid. On February 10-11, 2009, the Bill and Melinda Gates Foundation sponsored the meeting, "Mucosal Immunology of the Gut," to review the advances in the understanding of the gut immune system and to chart a course for the development of vaccines that can induce immunity in the intestines.

There are several reasons to develop mucosal vaccines. Most pathogens have a mucosal port of entry and although parenteral vaccination provides protection in some instances, in most cases a mucosal route of vaccination may be necessary. In addition, mucosal vaccines would allow immunization without nee- dles, reducing the risk of transmission of infections and speeding vaccine deployment. For instance, in 1996, 121 million children were immunized with the oral polio vaccine over a 2-day period.

A successful enteric-mucosal vaccine should favor adsorption by the gut epithelium, direct antigen to specialized antigen-presenting cells in the gut, induce $\mathrm{B}$ - and T-cell responses in desired regions of the gut, stimulate immunological memory, and avoid or prevent oral tolerance. Practical considerations include vaccine formulation, delivery route, and measurement of correlates of protection. Development of mucosal adjuvants suitable for humans will also be needed for most subunit vaccines. New research could also show why many oral vaccines are less protective in developing as compared with industrialized countries.
THE GUT MUCOSAL IMMUNE SYSTEM

The gastrointestinal mucosa protects the body against colonization and invasion by pathogens while preventing immune responses against undegraded food antigens.

Mucosal vaccines are appropriate for a number of pathogens. These include pathogens that are non-invasive, such as Vibrio cholerae and enterotoxigenic Escherichia coli (ETEC); pathogens that produce gut inflammation, such as Shigella and rotavirus; and pathogens that invade through the intestine and enter the systemic circulation, such as Salmonella typhi, polio virus, and human immunodeficiency virus. Although the first category may need a mucosally administered vaccine for adequate protection, especially in immunologically unprimed individuals such as infants and young children, the latter, more invasive infections can probably be prevented by vaccines given either mucosally or parenterally, or by a combination of such routes. Although 30 or more parenteral vaccines are licensed, only a handful of oral vaccines are licensed (see Table 1).

\section{“TROPICAL BARRIERS" TO ENTERIC VACCINES}

Many oral vaccines have performed poorly in developing countries when compared with industrialized countries, a finding attributed mainly to chronic environmental enteropathy (CEE), also called tropical enteropathy, characterized by disturbances of digestive and absorptive functions (see Table 2). Factors that may contribute to CEE include poor sanitation, intestinal flora overgrowth, and histological changes characterized by inflammation and blunting of small intestinal villi leading to malabsorption. Children living under extreme poverty 
Table 1 List of licensed mucosal vaccines against enteric infections

\begin{tabular}{l}
\hline Oral live attenuated typhoid vaccine (Vivotif Oral) \\
Oral inactivated cholera (Dukoral, Shancol) \\
Oral live attenuated cholera CVD 103-HgR (Orochol) \\
Oral live attenuated rotavirus (RotaTeq and ROTARIX) \\
Oral polio virus (OPV) \\
Note: A previous oral rotavirus vaccine (RotaShield) was withdrawn from the market due to adverse side \\
effects (intussusception). \\
Table 2 Several live oral vaccines have performed less well in developing as \\
compared with industrialized regions \\
\hline Polio virus (OPV) \\
Rotavirus vaccine (RotaShield, ROTARIX, RIT 4237 bovine vaccine) \\
Live attenuated cholera vaccine CVD 103-HgR (Orochol) \\
Shigella flexneri 2a SC602 vaccine
\end{tabular}

are especially sensitive. Metagenomic analyses are now underway to evaluate the role of the host microbiome in the development of CEE.

Other factors that might affect the performance of oral vaccines in developing countries include deficiencies of nutrients such as vitamin A (retinoic acid) and zinc, which can influence the response to oral adjuvants and vaccines by affecting discrete subpopulations of intestinal dendritic cells and T-cells; persistent activation of the gut innate immune system by infectious agents such as helminths; and concomitant viral and bacterial infections.

Neonates are born without gut flora but reach adult levels within the first year. Maternal influences are important for the response toward vaccines in neonates. Mothers pass protective cytokines and other immune factors to infants prenatally and through breast milk. Breast milk from mothers of low socio-economic status in developing countries contains high titers of antibodies to enteric pathogens that can interfere with vaccine "take."

Strategies for coping with CEE include co-administration of vaccines with agents that improve gut integrity, such as zinc, vitamin $\mathrm{A}$, and possibly probiotics. Withdrawal of breast milk for a few hours before oral vaccination, especially with live vaccines such as rotavirus vaccines, improves immunogenicity in developing countries. Treatment against helminths before immunization with oral cholera vaccine improved vaccine performance.

\section{NEONATAL GUT MUCOSAL IMMUNITY}

Clear functional differences exist between neonates, and older children and adults with regard to innate and adaptive immune responses. Neonates lack T cellindependent antibody responses, which become fully functional by 4 years. Neonatal $\mathrm{T}$ cells develop more slowly than adult $\mathrm{T}$ cells and multiple immunizations are required to induce protective immunity against $\mathrm{T}$ cell-dependent antigens. However, infants in developing countries seem to have more mature immune systems and respond earlier than same-age European infants, presumably because of more diverse and frequent exposure to microbial antigens.

In neonates, injected killed vaccines can induce long-term unresponsiveness. For example, whole-cell pertussis vaccine (DTP) given at birth induces persistent ( $>9$ months) $\mathrm{B}$-cell hyporesponsiveness against pertussis antigens. The live-attenuated vaccines Bacillus Calmette-Guérin (BCG) and oral polio vaccine (OPV) seem to be the only current vaccines that consistently induce protective immunity following a single immunization at birth. Essential questions such as when and how intestinal B- and T-cell responsiveness against enteric vaccines develops during early life in children from developing countries as compared with those from industrialized countries have not yet been systematically addressed.

\section{GUT MUCOSAL IMMUNE RESPONSES}

The nature and anatomic distribution of immune responses required to confer protection are pathogen-specific and depend on where in the gut the vaccine is taken up. Induction of immune responses in the small intestine takes place primarily in Peyer's patches where microfold (M) cells shuttle antigen to dendritic cells (DCs) and other antigen-presenting cells, which activate $\mathrm{T}$ and $\mathrm{B}$ cells. Instruction of $\mathrm{T}$ and $\mathrm{B}$ cells is followed by their migration through the blood and lymph to preferential locations in the small intestine and in remote mucosal tissues, such as glandular tissues of the mouth, breast, and lacrymal glands. Induction of immune responses in the large intestine is usually best achieved by local rectal instillation and takes place in solitary follicles covered with $\mathrm{M}$ cells in the rectal and colonic mucosae.

The migration of activated immune cells is governed by interactions between integrins or "homing receptors" and chemokine receptors on $\mathrm{B}$ and $\mathrm{T}$ cells and corresponding tissue-specific ligands or "addressins" and chemokines produced by mucosal endothelial and epithelial cells. DCs play a critical role in this process by programming mucosal $\mathrm{T}$ cells and possibly B cells to express specific homing receptors and by determining the nature of the effector $\mathrm{T}$ (inflammatory, regulatory, etc.) and B (IgA, IgG, or IgE) cell responses.

Different infectious and nutritional settings will translate into expression of different DC, T cell, and B cell subsets in the gut, which in turn will influence the response to adjuvants and vaccines. The normal response to most ingested antigens, such as food antigens, is "tolerance", to prevent inflammation while maintaining local IgA production. In contrast, pathogens and effective vaccines provide "danger signals" that induce immune responses. When inflammatory signals are present, DCs activate T-cell precursors of Th1, Th2, and/or Th17 lineages. Experience with many millions doses of oral vaccines support the view that vaccines are unlikely to break the already established food tolerance. When inflammatory signals are present, DCs are altered and consequently the nature of the 
mucosal response is altered. For instance, patients with inflammatory bowel disease show impaired oral tolerance. How local infection and inflammation will alter the response to oral adjuvants and vaccines remains to be determined to define better vaccine strategies.

\section{INDUCTION OF GUT IMMUNOLOGICAL MEMORY}

IgA antibody responses in the gut after disease or vaccination in humans last for only 6-12 months; longer lasting immunity instead depends on mucosal immunologic memory, which has been shown to persist for many years after oral cholera vaccination. For diarrheal diseases, it is essential that memory cells can respond rapidly to pathogen re-encounter before disease symptoms appear. Gut mucosal B-cell memory remains scarcely studied and methods to measure mucosal-IgA Bcell memory in humans have not yet been established.

\section{VACCINE ADJUVANTS AND INNATE IMMUNITY}

Several grams of secretory IgA are produced in the gut each day and seem to contribute to gut barrier functions against microbial attack. Recent data indicate that polyspecific sIgA may also play a role in preventing disease transmission in mice. More research needs to explore the role of polyspecific sIgA as adjuncts to highaffinity adaptive sIgA antibodies in herd protection.

Mucosal adjuvants direct and amplify innate and adaptive immune responses and consist of particulates, surfactants, microspheres, enterotoxins, and other microbial derivatives including Toll-like receptor (TLR) ligands, and endogenous human immunomodulators (e.g., IL-1). Although mucosal adjuvants are most efficient when co-delivered physically with antigens, so that both are taken up by same APCs, recent data indicate that certain adjuvants (LT, CT) when delivered transcutaneously before antigen application or injection at the same skin site can also induce mucosal immunity.

\section{FORMULATIONS}

Suitable oral-vaccine formulations should protect antigen identity against stomach acidity and degradation by digestive enzymes, and allow efficient penetration across epithelial barriers. Potential formulations include protective enteric coatings, micro- and nanoparticles, bioadhesive polymers, surfactants, liposomes, virus-like particles, and emulsions. Nanoparticles and nanoemulsions seem to be more successful than their micronsized counterparts, and the coupling of epithelial M cell-binding proteins, e.g., cholera toxin B subunit or M cell-specific monoclonal antibodies, either to nanoparticles or directly to vaccine antigens can further facilitate antigen uptake and immune stimulation. A major challenge is to devise formulations that enable delivery of vaccines to particular segments of the gut, e.g., small intestine vs. colon.

\section{VACCINE DELIVERY ROUTES}

Traditional routes of mucosal immunization include oral, nasal, rectal, and transcutaneous. Oral administration results in immune responses at the exposed mucosa, upper digestive tract, and small intestine and specifically glandularlinked systems, such as the gut-mammary axis. Nasal administration does not induce immune responses in the gut in humans. Rectal delivery elicits immune responses in the large intestine but not in the stomach or small intestine. Acceptance of infant rectal vaccination needs to be assessed.

Sublingual administration is a new approach that results in the induction of mucosal and systemic T-cell and antibody responses with a broad dissemination to different mucosae, including the gastrointestinal and respiratory tracts, and the genital mucosa. Sublingual administration of an influenza vaccine protected mice against infection for one to two years and other sublingual vaccinations have resulted in protective gut-IgA and T-cell responses against Helicobacter pylori infection. Transcutaneous administration is another promising route that can elicit specific cellular and humoral responses systemically and in the mucosae. Parenteral administration might be used in tandem with oral vaccines, either as a prime or more likely as a boost; e.g., parenteral polio or cholera boosting stimulates antigen-specific sIgA responses in naturally primed individuals. Vaccine-induced serum IgG can also protect against intestinal pathogens either by preventing subepithelial microbial spread (e.g., shigellosis) or by invasion through draining vessels (e.g., typhoid).

\section{SURROGATES AND CORRELATES OF PROTECTION}

Gut immune response measurements include assays for antigen-specific IgA (or IgG or IgM) antibody-secreting cells from intestinal biopsies, as well as less invasive measurements of antigen-specific IgA and $\operatorname{IgG}$ in gastrointestinal lavages or fecal extracts, and gut-derived $\mathrm{B}$ - and $\mathrm{T}$ cell responses in blood. However, most of these measurements are surrogates rather than correlates of protection and only show that an immune response is taking place. Correlates of protection will vary for each vaccine and may be based on toxin or virus neutralization, bactericidal antibodies, inhibition of invasion or adherence, or detection of cytotoxic cells ideally at the gut mucosal effector sites. Existing techniques need to be made less invasive and more suitable for being used with small sample volumes from infants and young children. To date, none of these approaches have obtained regulatory acceptance limiting their usefulness to support licensure of new vaccines.

\section{ANIMAL MODELS}

Animal models are valuable tools, however significant differences exist in the mucosa of humans and that of other species. For example and in contrast to humans, mice do not have tonsils and their sublingual mucosa is keratinized, a large portion of serum IgA in mice is polymeric and transported through the bile into the small intestine, and only one $\operatorname{IgA}$ isotype is found in mice. Species-specific differences make non-human primates a less than ideal model. A "humanized mouse" now under development may offer new opportunities. Humans remain the ideal testing population.

\section{THE ROAD FORWARD}

To explore chronic environmental enteropathy in relation to vaccines, studies should be conducted with licensed vaccines, both oral killed and live bacterial 
and viral vaccines and for comparisons also live and killed parenteral vaccines (e.g., OPV and inactivated polio vaccine (IPV)) in developing countries. Sorely needed are field-ready, standardized, and validated non-invasive assays that do not require large sample volumes, and reference reagents. Research is needed to understand the mechanisms of adaptive B-cell and T-cell memory in the gut. A better knowledge of gut immune responsiveness during early life is required to establish the usefulness of mucosal vaccination against enteric pathogens encountered by neonates and young infants from developing and industrialized countries.

With a concerted research agenda, mucosal immunologists may accelerate development of life-saving enteric vaccines that can reduce the burden of disease and mortality in developing countries and worldwide.

The viewpoints expressed in this article do not necessarily reflect those of other attendants/speakers at the meeting. The speakers were:

Jan Agosti, Bill \& Melinda Gates Foundation
Carl R Alving, Walter Reed Army Institute of Research

A Louis Bourgeois, Program for Appropriate Technology in Health

Thomas Brewer, Bill \& Melinda Gates Foundation

John Clements, Tulane University School of Medicine

Cecil Czerkinsky, International Vaccine Institute

Jan Holmgren, University of Gothenburg

Bana Jabri, University of Chicago

Hiroshi Kiyono, University of Tokyo

Tobias Kollmann, University of British

Columbia

Myron M Levine, University of Maryland

Gareth Morgan, Swansea University

Marian Neutra, Harvard Medical

School

Derek Thomas O'Hagan, Novartis

Steven Reed, Infectious Disease

Research Institute

Hans-Christian Reinecker, Harvard Medical School

John B Robbins, National Institute of Child Health and Human Development, $\mathrm{NIH}$
Juan Antonio Solon, British Medical Research Council

Marcelo Sztein, University of Maryland

Richard Ward, Cincinnati Children's Hospital

Howard L Weiner, Harvard Medical School

Chris B Wilson, University of Washington

\section{DISCLOSURE}

The authors declared no conflict of interest.

\section{ACKNOWLEDGMENTS}

The authors wish to thank the Bill \& Melinda Gates Foundation for funding the meeting and supporting mucosal vaccine research. The authors would also like to acknowledge the contribution of the International Vaccine Institute (IVI), an international nonprofit organization dedicated to vaccine development for the people in developing countries, and the contributions of the scientists and support staff at the Bill \& Melinda Gates Foundation, especially Jan Agosti and Gretchen Meller for organizing the meeting and Emily Wright and Zoey Diaz for logistical support. Finally, they acknowledge Catherine Zandonella for assistance in preparing this paper.

C) 2009 Society for Mucosal Immunology 